\title{
Reoperation rates after Ex-PRESS versus trabeculectomy for primary open-angle or normal-tension glaucoma: a national database study in Japan
}

\author{
Yohei Hashimoto $\mathbb{B}^{1,2} \cdot$ Nobuaki Michihata $^{3} \cdot$ Hiroki Matsui ${ }^{2} \cdot$ Kiyohide Fushimi $^{4} \cdot$ Hideo Yasunaga $^{2} \cdot$ \\ Makoto Aihara (1D ${ }^{1}$
}

Received: 27 March 2019 / Revised: 28 August 2019 / Accepted: 9 October 2019 / Published online: 23 October 2019

(c) The Author(s), under exclusive licence to The Royal College of Ophthalmologists 2019

\begin{abstract}
Objective To compare reoperation rates between Ex-PRESS implantation and trabeculectomy.

Methods We performed a retrospective cohort study using a national inpatient database in Japan. Patients aged 18-85 years with primary open-angle glaucoma or normal-tension glaucoma who underwent Ex-PRESS or trabeculectomy from 2010 to 2017 were included. We compared the reoperation rates between Ex-PRESS and trabeculectomy using mixed effects cox regression models. Covariates were sex, age, diabetes mellitus, simultaneous cataract surgery, ocular surgical history, and annual hospital volume for glaucoma surgery. Furthermore, we conducted propensity score (PS) matching and instrumental variable (IV) analyses to confirm the results of the conventional cox regression. We also compared total hospitalisation costs between the two treatments in the PS-matched groups.

Results In total, 1027 eyes underwent Ex-PRESS and 6910 eyes underwent trabeculectomy. The reoperation rates were 7.6\% and 5.8\% in the Ex-PRESS and trabeculectomy groups, respectively. The most frequently performed type of reoperation was trabeculectomy in both groups. The mixed effects cox regression showed that Ex-PRESS had significantly higher reoperation rates than trabeculectomy [adjusted hazard ratio (aHR), 1.72; 95\% confidence interval (CI), 1.31-2.25; $p$ $<0.001$ ]. The PS and IV analyses also showed similar results (for PS analysis: aHR, 2.13; 95\% CI, 1.45-3.13; $p<0.001$; for IV analysis: aHR, 2.26; 95\% CI, 1.05-4.85; $p=0.037$ ). The total hospitalisation cost of Ex-PRESS (US\$7076) was significantly greater than that of trabeculectomy (US\$6223) $(p<0.001)$.

Conclusion Ex-PRESS implantation had significantly higher reoperation rates and greater cost than trabeculectomy.
\end{abstract}

\section{Introduction}

The aim of glaucoma surgery is to reduce the intraocular pressure (IOP) and prevent visual field progression. The gold standard of glaucoma surgery is trabeculectomy. This

Yohei Hashimoto

youhashimoto-tky@umin.ac.jp

1 Department of Ophthalmology, Graduate School of Medicine, The University of Tokyo, Tokyo, Japan

2 Department of Clinical Epidemiology and Health Economics, School of Public Health, The University of Tokyo, Tokyo, Japan

3 Department of Health Services Research, Graduate School of Medicine, The University of Tokyo, Tokyo, Japan

4 Department of Health Policy and Informatics, Tokyo Medical and Dental University Graduate School, Tokyo, Japan procedure has been used since the 1960s [1], although several new procedures that lower the IOP have been developed in recent years [2]. One is implantation of the ExPRESS (Alcon, Fort Worth, TX, USA), an external minishunt that is used worldwide [3-6]. The Ex-PRESS is inserted at the limbus under a partial-thickness scleral flap to filtrate the aqueous humour to the subconjunctival space. Ex-PRESS implantation is a modification of trabeculectomy and does not require sclerectomy or iridectomy. Ex-PRESS implantation was shown to shorten the duration of anterior chamber opening and reduce early postoperative complications such as hyphaema, inflammation (flare), and hypotony compared with trabeculectomy [7-9].

To date, six randomised controlled trials (RCTs) [7, 9-13] have compared the efficacy and safety between Ex-PRESS implantation and trabeculectomy. Some of these studies showed significant differences in the success rate (achievement of adequate IOP, no subsequent need for prescription of 
IOP-lowering medication, and no further surgery performed for glaucoma control) in favour of Ex-PRESS implantation, but others showed no significant difference. Two metaanalyses summarising the RCTs indicated that Ex-PRESS implantation and trabeculectomy had similar efficacy in lowering the IOP and qualified success rate but that ExPRESS was more likely to achieve complete success [8, 14].

However, none of these RCTs defined the success rate solely as reoperation for glaucoma. Therefore, whether ExPRESS or trabeculectomy is superior in terms of reoperation rates remains unknown. Reoperations place a physiological and psychological burden on patients and increase the difficulty of the surgery itself [15]. Thus, we compared the reoperation rates between Ex-PRESS implantation and trabeculectomy using a nationwide inpatient database in Japan. We also compared total hospitalisation costs between the two treatments.

\section{Patients and methods}

\section{Data source}

We conducted a large-scale retrospective cohort study using the diagnosis procedure combination (DPC) database from July 2010 to March 2017. All 82 academic hospitals in Japan are required to take part in the database, and participation by community hospitals is voluntary. The database includes the following information: (1) patient characteristics (age, sex, body weight, and height); (2) dates of hospitalisation; (3) unique identifiers of hospitals; (4) diagnoses, comorbidities, at admission, and complications after admission recorded with text data in Japanese and the International Classification of Diseases, Tenth Revision codes; (5) procedures coded with Japanese original codes; (6) laterality of surgery; and (7) discharge destination (the same hospital, another hospital, or nursing care facilities). The DPC database includes inpatient data from more than 1000 hospitals. Approximately 300 of these hospitals also have outpatient data. The validity of diagnoses and procedure records in the DPC database has been reported in a previous study [16].

The need for informed consent was waived because of the anonymous nature of the data. The Institutional Review Board of The University of Tokyo approved this study.

\section{Patient selection}

We identified patients with primary open-angle glaucoma (POAG) or normal-tension glaucoma (NTG) who were admitted to undergo Ex-PRESS implantation or trabeculectomy. Note that we only included the 300 hospitals with outpatient data because a postoperative follow-up period was needed to perform the survival analysis. We counted patients who underwent bilateral operations within the same admission as one right and one left operation. The end of the follow-up period was defined as the date of the last visit. We excluded data on eyes with missing data on laterality, eyes that underwent more than one operation for glaucoma during the same admission, and eyes that underwent simultaneous surgery of the vitreous and retina. If a patient was admitted for reoperation for glaucoma in the same eye more than once during the observation period, we included only the data of the first admission for analysis. Finally, we excluded patients aged $>85$ or $<18$ years.

\section{Variables}

The baseline variables were sex, age, operative laterality, diabetes mellitus (DM), simultaneous cataract surgery, history of cataract surgery, history of vitreous and retina surgery, and annual hospital volume for glaucoma surgery (Ex-PRESS implantation and trabeculectomy).

We selected DM as one of the covariates because several previous observational studies have shown that among patients with OAG, those with DM have a poorer outcome after trabeculectomy than those without DM [17-19]. We also selected simultaneous cataract surgery as a covariate because cataract surgery performed on the same day as glaucoma surgery was shown to reduce the hazard ratio (HR) for adverse outcomes [20]. We selected a history of cataract, vitreous, and retina surgery as covariates based on a previous study that assessed a history of intraocular surgery [21]. Hospital volume was defined as the average number of glaucoma surgeries performed at each hospital annually and was categorised into quartiles so that the number of patients in each group was almost equal.

The primary outcome was reoperation for glaucoma (ExPRESS implantation, trabeculectomy, aqueous shunt to extraocular reservoir, cyclophotocoagulation, or cyclocryotherapy). Small interventions such as needling procedures were not regarded as a reoperation. The time to reoperation was calculated from the date of the first operation (Ex-PRESS implantation or trabeculectomy) to the date of reoperation for glaucoma. Patients who did not undergo a reoperation during the follow-up period were considered to be censored at the date of the last visit.

The secondary outcomes were total hospitalisation costs and length of hospital stay. The total costs included costs for surgery, anaesthesia, medication, examination, diet, nursing care, and the basic hospital fee. The length of stay was defined as the period between admission and discharge.

\section{Statistical analysis}

The chi-square test was used to compare the proportions of categorical variables such as sex, and the $t$-test was used to compare the average of continuous variables such as age. 
Kaplan-Meier survival analysis was used to calculate the incidence proportion of reoperation between the two groups (Ex-PRESS implantation and trabeculectomy), and the log-rank test was used to compare the two survival curves. We used a mixed effects cox regression model to adjust for relevant explanatory variables and the two clusters: eyes (right or left) within patients and patients within hospitals. In this model, we treated the receipt of Ex-PRESS implantation, sex, age, DM, simultaneous cataract surgery, history of cataract surgery, history of vitreous and retina surgery, and annual hospital volume as fixed effects and treated patients and hospitals as random effects.

\section{Propensity score (PS) analysis}

To increase the robustness of the above-described cox regression model, we performed PS analysis. We calculated the PS score using a logistic model in which the receipt of Ex-PRESS implantation was regressed against the covariates (sex, age, DM, simultaneous cataract surgery, history of cataract surgery, history of vitreous and retina surgery, and annual hospital volume). We performed one-to-one matching of patients between the Ex-PRESS and trabeculectomy groups with the closest estimated PS. We set the calliper width at $20 \%$ of the standard deviation and used nearestneighbour matching without replacement. We compared the baseline covariates between the two treatments after matching using the absolute standardised difference, with values of $>10 \%$ considered to indicate a significant imbalance. We then compared the time to reoperation between the Ex-PRESS and trabeculectomy groups in the PSmatched patients using the mixed effects cox regression model in which the receipt of Ex-PRESS implantation was treated as a fixed effect and the two clusters (eyes within patients and patients within hospitals) were treated as random effects. We further investigated the potential for residual confounding by calculating the E-value [22].

\section{Instrumental variable (IV) analysis}

We also performed an IV analysis because conventional multivariable cox regression and PS analyses cannot remove hidden biases caused by unmeasured confounders. IV analysis has the potential to remove unmeasured confounding. The baseline characteristics including unmeasured variables become balanced between the two treatments, allowing us to mimic an RCT [23]. IV analysis requires two assumptions [24]: (1) the IV is strongly associated with the treatment (in our case, Ex-PRESS implantation or trabeculectomy), and (2) the IV is not associated with the outcome (reoperation) except through its effect on the treatment.

If each hospital has its own selection criteria for which of the two treatments (Ex-PRESS implantation or trabeculectomy) should be performed regardless of patient characteristics, the treatment selection is more likely to be determined based on hospital preference rather than patient characteristics [25-28]. Under these conditions, the proportion of Ex-PRESS implantations performed at each hospital can be regarded as an IV. In our IV analysis, we excluded the hospitals with an annual hospital volume of $\leq 10$. We calculated the proportion of Ex-PRESS implantations performed at each hospital by dividing the number of eyes treated with ExPRESS implantation by the total number of eyes treated with either Ex-PRESS implantation or trabeculectomy.

We used two-stage residual inclusion estimation for the IV analysis [24, 25, 29]. In the first-stage model, we analysed the association between receipt of Ex-PRESS implantation and the IV (the proportion of Ex-PRESS implantations performed) with adjustment for patient- and eye-level covariates, and we then predicted the probability of receiving Ex-PRESS implantation. Next, we obtained the raw residual for each eye by calculating the difference between the predicted probability and the actual Ex-PRESS implantations received.

In the second-stage model, we estimated the association between treatment and the time to reoperation using a mixed effects cox regression model. In this model, the receipt of Ex-PRESS implantation, sex, age, DM, simultaneous cataract surgery, history of cataract surgery, history of vitreous and retina surgery, and the residuals calculated in the first stage were treated as fixed effects, and patients and hospitals were treated as random effects.

We also compared the average total costs and length of stay between Ex-PRESS implantation and trabeculectomy in the PS-matched groups using a $t$-test.

For the sensitivity analysis, we further performed the following two analyses. (1) We excluded the patients whose discharge destination was not the same hospital because they may cause informative censoring. (2) We considered two "worst-case" violations of the independence assumption: one scenario in which all patients who were censored are assumed to undergo reoperation, and the other in which all patients who were censored survive as long as the longest survival time observed in the study.

We used a significance level of $p<0.05$ for all statistical tests, and all reported $p$ values were two sided. We used the statistical programming language $\mathrm{R}(\mathrm{R}$ version 3.5.0, $\mathrm{R}$ Project for Statistical Computing) for all statistical analyses.

\section{Results}

We identified 7937 eligible eyes (1027 treated by ExPRESS implantation and 6910 treated by trabeculectomy). The PS matching produced 1025 pairs (Fig. 1). 
Table 1 shows the patient and eye characteristics. Patients in the Ex-PRESS group were significantly older and more likely to have a surgical history than patients in the trabeculectomy group. The mean follow-up period was 1.7 years (maximum, 4.8 years) in the Ex-PRESS group and 2.0 years (maximum, 6.7 years) in the trabeculectomy group. After PS matching, the characteristics between the two treatments were closely balanced.

Table 2 shows the types of reoperations performed for glaucoma in each primary surgery. The most frequently

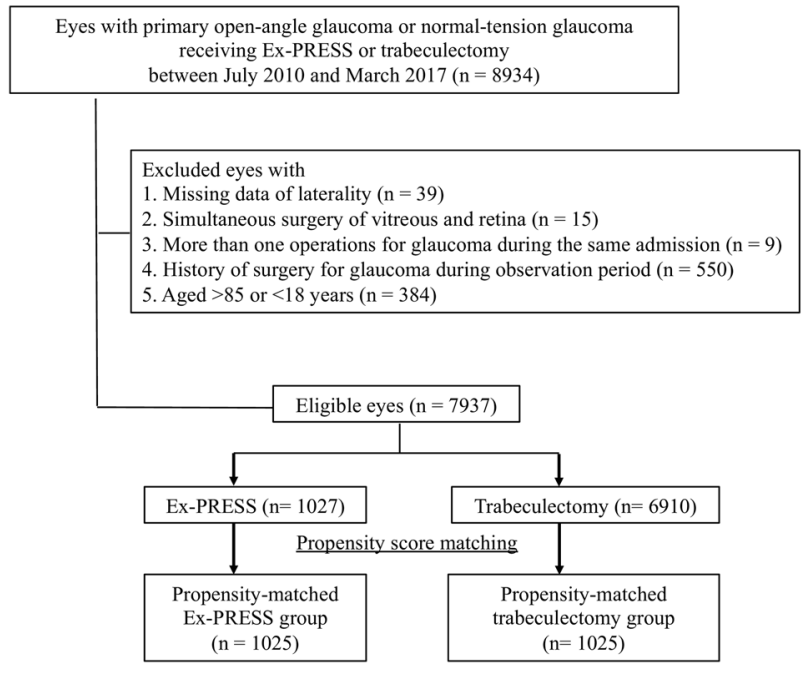

Fig. 1 Patient selection performed reoperation was trabeculectomy in both groups (6.2\% in the Ex-PRESS group and 5.2\% in the trabeculectomy group). No tube shunting, cyclophotocoagulation, or cyclocryotherapy was performed in the reoperations for glaucoma.

Figure 2 shows the results of the Kaplan-Meier analysis comparing the rates of reoperation between the Ex-PRESS and trabeculectomy groups. The 4-year incidence proportion of reoperation was $13.2 \%$ in the Ex-PRESS group and $9.8 \%$ in the trabeculectomy group. The Ex-PRESS group had a significantly higher rate of reoperation than the trabeculectomy group $(p<0.001, \log$-rank test).

Table 3 shows the results of the mixed effects cox regression analysis for reoperation for glaucoma. The ExPRESS group had a significantly higher rate of reoperation than the trabeculectomy group [adjusted HR, 1.72; 95\% confidence interval (CI), 1.31-2.25; $p=0.001]$. Male sex, younger age, and glaucoma surgery alone (without simultaneous cataract surgery) were significantly associated with an increase in the rate of reoperation for glaucoma. History of cataract surgery, history of vitreous and retina surgery, and the annual hospital volume did not show a significant association with reoperation for glaucoma.

The PS matching analysis showed that the rate of reoperation was significantly higher in the Ex-PRESS than trabeculectomy group (adjusted HR, 2.13; 95\% CI, $1.45-3.13 ; p<0.001)$. The $E$-value was calculated as 3.68 (lower limit of $95 \% \mathrm{CI}, 2.26$ ).

Table 1 Patient and ophthalmic characteristics

\begin{tabular}{|c|c|c|c|c|c|c|}
\hline & \multicolumn{3}{|l|}{ Unmatched } & \multicolumn{3}{|c|}{ Propensity score matched } \\
\hline & Ex-PRESS & Trabeculectomy & $\begin{array}{l}\text { Standardized } \\
\text { difference }(\%)\end{array}$ & Ex-PRESS & Trabeculectomy & $\begin{array}{l}\text { Standardized } \\
\text { difference }(\%)\end{array}$ \\
\hline No. of patients & 881 & 5531 & & 879 & 994 & \\
\hline Male & $511(58.0)$ & $3165(57.2)$ & 1.6 & $509(57.9)$ & $576(57.9)$ & 0.1 \\
\hline Age, years & $68.7 \pm 10.5$ & $66.8 \pm 11.4$ & 17.2 & $68.7 \pm 10.5$ & $68.7 \pm 10.7$ & 0.1 \\
\hline Diabetic mellitus & $172(19.5)$ & $843(15.2)$ & 11.3 & $171(19.5)$ & $196(19.7)$ & 0.7 \\
\hline No. of eyes & 1027 & 6910 & & 1025 & 1025 & \\
\hline Operative laterality, left & $520(50.6)$ & $3469(50.2)$ & 0.9 & $520(50.7)$ & $497(48.5)$ & 4.5 \\
\hline \multicolumn{7}{|l|}{ History of other surgeries } \\
\hline Cataract & $108(10.5)$ & $305(4.4)$ & 23.4 & $106(10.3)$ & $111(10.8)$ & 1.6 \\
\hline Vitreous and retina & $39(3.8)$ & $62(0.9)$ & 19.2 & $37(3.6)$ & $36(3.5)$ & 0.5 \\
\hline $\begin{array}{l}\text { Simultaneous cataract } \\
\text { surgery }\end{array}$ & $258(25.1)$ & 1965 (28.4) & 7.5 & $258(25.2)$ & $262(25.6)$ & 0.9 \\
\hline Annual hospital volume & & & 54.5 & & & 4.3 \\
\hline Low $(<52)$ & $408(39.7)$ & $1540(22.3)$ & & $406(39.6)$ & $401(39.1)$ & \\
\hline Middle (52-115) & $352(34.3)$ & $1861(26.9)$ & & $352(34.3)$ & $353(34.4)$ & \\
\hline High (115-184) & $154(15.0)$ & $1918(27.8)$ & & $154(15.0)$ & $167(16.3)$ & \\
\hline Very high $(>184)$ & $113(11.0)$ & $1591(23.0)$ & & $113(11.0)$ & $104(10.1)$ & \\
\hline Follow-up period, years & $1.7 \pm 1.3$ & $2.0 \pm 1.8$ & 18.6 & $1.7 \pm 1.3$ & $2.2 \pm 1.8$ & 28.9 \\
\hline
\end{tabular}

Data are presented as $n(\%)$ or mean \pm standard deviation 
Table 2 Relationship between types of primary surgery and reoperation

\begin{tabular}{llll}
\hline & \multicolumn{2}{l}{ Primary surgery } \\
\cline { 2 - 4 } & $\begin{array}{l}\text { Ex-PRESS } \\
(n=1027)\end{array}$ & $\begin{array}{l}\text { Trabeculectomy } \\
(n=6910)\end{array}$ & $\begin{array}{l}\text { Total } \\
(n=7937)\end{array}$ \\
\hline $\begin{array}{l}\text { Reoperation } \\
\text { Ex-PRESS }\end{array}$ & $14(1.4)$ & $40(0.6)$ & $54(0.7)$ \\
Trabeculectomy & $64(6.2)$ & $361(5.2)$ & $425(5.4)$ \\
\hline
\end{tabular}

Data are presented as $n(\%)$

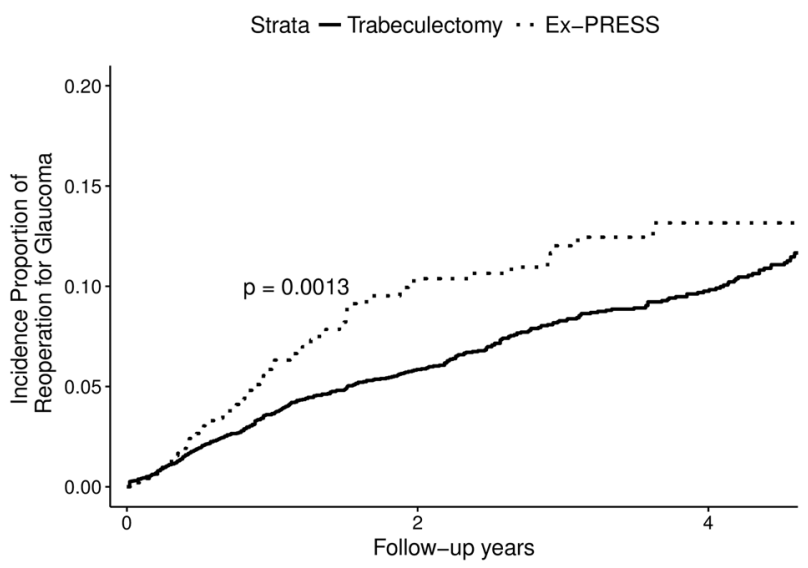

Fig. 2 Kaplan-Meier curves of the incidence proportion of reoperation for glaucoma in Ex-PRESS implantation vs. trabeculectomy. The proportions of reoperation at $1,2,3$, and 4 years were $6.1 \%, 10.2 \%$, $11.8 \%$, and $12.9 \%$, respectively, in the Ex-PRESS group and $3.6 \%$, $5.8 \%, 8.3 \%$, and $9.8 \%$, respectively, in the trabeculectomy group

In the IV analysis, the $F$-statistic indicated that the proportion of Ex-PRESS implantations was a sufficiently strong IV for prediction of Ex-PRESS use $(F=1132, p<$ $0.001)$. The rate of reoperation was significantly higher in the Ex-PRESS than trabeculectomy group (adjusted HR, 2.26; 95\% CI, 1.05-4.85; $p=0.037$ ).

The average total hospitalisation cost of the Ex-PRESS [7076 US dollars (USD) $(1 \mathrm{USD}=112.5$ Japanese yen)] was significantly greater than that of the trabeculectomy (US\$6223) in the PS-matched groups $(p<0.001)$. The breakdown of the total cost in the Ex-PRESS and trabeculectomy group was: (1) surgery and anaesthesia (3920 vs. US\$2944, respectively; $p<0.001$ ), (2) basic hospital fees (US\$2643 vs. US $\$ 2725$, respectively; $p=0.15$ ), and (3) others such as diet, medication, and examination (US\$513 vs. US\$554, respectively; $p=0.044$ ). The length of hospital stay of the Ex-PRESS group was significantly shorter than that of the trabeculectomy group (12.4 vs. 13.1 days, respectively; $p=0.025$ ).

For the sensitivity analysis in which we excluded the patients whose discharge destination was not the same hospital as that to which they were admitted $(n=374)$, the
Table 3 Mixed effects cox regression analysis predicting reoperation for glaucoma

\begin{tabular}{llll}
\hline & Hazard ratio & $\begin{array}{l}95 \% \text { confidence } \\
\text { interval }\end{array}$ & $p$ \\
\hline $\begin{array}{l}\text { Type of primary surgery } \\
\text { Trabeculectomy }\end{array}$ & $\begin{array}{l}\text { Reference } \\
\text { Ex-PRESS }\end{array}$ & & \\
Male (vs. female) & 1.72 & $1.31-2.25$ & 0.001 \\
Age (years) & 0.99 & $1.01-1.50$ & 0.044 \\
Diabetes mellitus & 1.16 & $0.98-1.00$ & 0.039 \\
Surgical history & & & 0.23 \\
Cataract & 1.31 & $0.85-2.02$ & 0.23 \\
Vitreous and retina & 0.32 & $0.10-1.05$ & 0.060 \\
Simultaneous cataract & 0.76 & $0.60-0.97$ & 0.026 \\
surgery & & & \\
Annual hospital volume & & & \\
Low (<52) & Reference & & 0.35 \\
Middle (52-115) & 0.85 & $0.61-1.19$ & 0.13 \\
High (115-184) & 1.34 & $0.92-1.96$ & 0.28 \\
Very high (>184) & 1.32 & $0.79-2.21$ & \\
\hline
\end{tabular}

HRs were consistent with the original ones. For the other sensitivity analysis, in which all patients who were censored were assumed either to undergo reoperation or to survive as long as the longest survival time observed in the study, the HRs were also consistent with the original ones.

\section{Discussion}

This study showed that Ex-PRESS implantation was associated with a significantly higher rate of reoperation than trabeculectomy with control for other factors. The PS matching and IV analyses confirmed the results of the conventional cox regression model. Ex-PRESS was also more expensive than trabeculectomy.

There are four previous RCTs [9-12] comparing ExPRESS implantation and trabeculectomy, but they did not treat reoperation rates as the primary outcome. To the best of our knowledge, this is the first study to focus mainly on the time to reoperation for glaucoma. Reoperation is relatively rare $(<10 \%)$ as shown in our study; thus, many patients are needed for comparative analysis. However, this is difficult to perform in RCTs. A nationwide database in our study enabled us to use a large sample size than the previous RCTs $(n=30-120)$ and to draw a more reliable inference.

Previous meta-analyses [8, 14] showed either better outcomes in the Ex-PRESS group than trabeculectomy group or identical outcomes between the two groups. Their trends in favour of Ex-PRESS are inconsistent with ours. 
This discrepancy may be attributed to racial difference; most of the patients included in our study were probably Asians. One radial difference is the difference in the tendency toward scarring. Asians reportedly have a higher tendency to develop dermal hyperpigmentation and scarring [30, 31]. The eye tissue of Asians may also have this characteristic. In fact, a previous study showed that Asians tended to have poorer success rates of trabeculectomy than whites; one cause may be excessive fibrosis at the subconjunctival space. Furthermore, implanted biomaterials generally elicit a significant reaction from the host, known as the foreign body response [32]. Ex-PRESS is a biomaterial and thus might have led to a stronger foreign body response in Asians than in Caucasians. Another racial difference is the difference in the size of the anterior segment. Asian eyes have smaller anterior segments than Caucasian eyes [33]. Asian eyes thus may have more frequent contact of the Ex-PRESS device onto the iris surface at the inserted angle than Caucasian eyes. Sustained contact of this device would induce chronic iris pigment dispersion and subsequent inflammation or disruption of the blood-aqueous barrier. These racial differences may have caused the higher reoperation rate for Ex-PRESS than for trabeculectomy in the current study. Our findings thus may be limited to Asians.

When comparing the two treatments, we cannot ignore the cost issue. In our study, although the length of stay was shorter in Ex-PRESS than in trabeculectomy, ExPRESS implantation was more costly in total than trabeculectomy. Looking at the breakdown, there was no significant difference in basic hospital fees between the two treatments, but cost of surgery was higher in ExPRESS than in trabeculectomy. In Japan, the medical service fee of Ex-PRESS implantation is set higher than that of trabeculectomy because of the cost of the device itself; thus, our result is not unexpected. A previous RCT showed that Ex-PRESS implantation was associated with greater costs than trabeculectomy at 1 year after surgery [34]. In their study, the greater cost in the Ex-PRESS group was mainly due to the device itself, and there was no difference in the cost of follow-up visits, additional procedures performed, or topical medications administered between the two treatments. Another study also showed that Ex-PRESS implantation was 4.9 times more expensive than trabeculectomy [35]. These results suggest that Ex-PRESS may be less cost effective, but this remains to be elucidated.

Male sex, younger age, and glaucoma surgery alone were also significantly associated with higher rates of reoperation for glaucoma in our study. Regarding sex, several previous studies have shown that sex hormones such as oestrogen and progestin might play a role in protection of the optic nerve $[36,37]$. This hypothesis may also be applied to our finding that men had a higher rate of reoperation. Regarding age, the Advanced Glaucoma Intervention Study showed that younger age was significantly associated with the failure of trabeculectomy [19], and this is consistent with our results. Regarding simultaneous cataract surgery, a previous systematic review reported that trabeculectomy alone lowers long-term IOP more than combined cataract extraction [38]. Our results are not in line with these results. This discrepancy may be explained partly by the fact that the studies included in the systematic review were published before 2000. Recent improvements in the surgical techniques and instruments for cataract surgery may have contributed to the good outcome of simultaneous surgery in our results. Indeed, more recently, another database study showed that combined cataract surgery and trabeculectomy was significantly associated with a lower rate of subsequent operations for glaucoma [20]. Their findings support our results.

Several limitations in our study should be acknowledged. First, the DPC database does not provide the details of ophthalmologic examinations including visual field, IOP, and the number of glaucoma medications. Thus, we could not adjust for the severity of glaucoma at baseline, which may have led to unmeasured confounding. Even PS analysis cannot cope with this problem. To minimise this limitation, we conducted the IV analysis as a confirmatory analysis of the conventional cox regression model. Furthermore, the lower limit of the CI of the $E$-value was 2.26. This means that to make the $95 \% \mathrm{CI}$ of HR include the null (that is, make the causality disappear), an unmeasured confounder has to be associated both with treatment (Ex-PRESS or trabeculectomy) and outcome (reoperation) by a ratio of 2.26-fold each. Considering that a review study showed that there are no proven indications for favouring Ex-PRESS implantation [5], there may be no stronger confounders over 2.26 times at least in terms of the treatment selection. Thus, the evidence for causality looks reasonably strong. Second, various types of surgical techniques were included such as those with and without mitomycin $\mathrm{C}$ and its different concentrations and durations. Third, attrition bias will occur for reasons such as loss to follow-up, transfer to another hospital, and death. We thus performed two sensitivity analyses, which showed consistent results with the original ones. Fourth, the reoperation rate is a relatively soft endpoint rather than a hard one such as the success rate; the criteria used to consider the previous surgery failed, and to choose the reoperation varies from one hospital to another.

Despite these limitations, our study is advantageous in that we used a large real-world national database. Unlike small observational studies in which surgeon- and hospitalspecific factors may have a large impact, our study has high external validity. Furthermore, we drew a reliable inference regarding rare events such as reoperation. 
In conclusion, our study showed that the rate of reoperation for glaucoma was significantly higher in ExPRESS implantation than in trabeculectomy in patients with POAG or NTG. We should consider possibility of reoperation and greater costs when choosing Ex-PRESS implantation.

\section{Summary}

\section{What was known before}

- Ex-PRESS and trabeculectomy reportedly have similar efficacy and safety profiles. However, whether ExPRESS or trabeculectomy is superior in terms of reoperation rates remains unknown.

\section{What this study adds}

- Ex-PRESS had a higher rate of reoperation for glaucoma than trabeculectomy. Ex-PRESS had greater cost during admission than trabeculectomy.

Funding This work was supported by grants from the Ministry of Health, Labour and Welfare, Japan (H30-Policy-Designated-004 and H29-ICT-General-004) and the Ministry of Education, Culture, Sports, Science and Technology, Japan (17H04141)

\section{Compliance with ethical standards}

Conflict of interest The authors declare that they have no conflict of interest.

Publisher's note Springer Nature remains neutral with regard to jurisdictional claims in published maps and institutional affiliations.

\section{References}

1. Cairns JE. Trabeculectomy. Preliminary report of a new method. Am J Ophthalmol. 1968;66:673-9.

2. Higginbotham EJ, Alexis D. Is newer necessarily better? The evolution of incisional glaucoma surgery over the last 100 years. Am J Ophthalmol. 2018;191:25-9.

3. Arora KS, Robin AL, Corcoran KJ, Corcoran SL, Ramulu PY. Use of various glaucoma surgeries and procedures in medicare beneficiaries from 1994 to 2012. Ophthalmology. 2015;122:1615-24.

4. Sugiyama T, Shibata M, Kojima S, Ueki M, Ikeda T. The first report on intermediate-term outcome of Ex-PRESS ${ }^{\circledR}$ glaucoma filtration device implanted under scleral flap in Japanese patients. Clin Ophthalmol. 2011;5:1063-6.

5. Moisseiev E, Zunz E, Tzur R, Kurtz S, Shemesh G. Standard trabeculectomy and Ex-PRESS miniature glaucoma shunt: a comparative study and literature review. J Glaucoma. 2015;24:410-6.

6. Zhang M, Li B, Sun Y. EX-PRESS and ahmed glaucoma valve in treatment of refractory glaucoma. Acta Ophthalmol. 2016;94:e382-3.
7. Arimura S, Takihara Y, Miyake S, Iwasaki K, Gozawa M, Matsumura $\mathrm{T}$, et al. Randomized clinical trial for early postoperative complications of Ex-PRESS implantation versus trabeculectomy: complications postoperatively of Ex-PRESS versus trabeculectomy study (CPETS). Sci Rep. 2016;6:1-7.

8. Wang W, Zhang X. Meta-analysis of randomized controlled trials comparing EX-PRESS implantation with trabeculectomy for open-angle glaucoma. PLoS ONE. 2014;9:e100578.

9. Dahan E, Ben Simon GJ, Lafuma A. Comparison of trabeculectomy and Ex-PRESS implantation in fellow eyes of the same patient: a prospective, randomised study. Eye. 2012;26:703-10.

10. Gonzalez-Rodriguez JM, Trope GE, Drori-Wagschal L, Jinapriya D, Buys YM. Comparison of trabeculectomy versus Ex-PRESS: 3-year follow-up. Br J Ophthalmol. 2016;100:1269-73.

11. de Jong L, Lafuma A, Aguadé AS, Berdeaux G. Five-year extension of a clinical trial comparing the EX-PRESS glaucoma filtration device and trabeculectomy in primary open-angle glaucoma. Clin Ophthalmol. 2011;5:527-33.

12. Netland PA, Sarkisian SR, Moster MR, Ahmed IIK, Condon G, Salim S, et al. Randomized, prospective, comparative trial of express glaucoma filtration device versus trabeculectomy (XVT Study). Am J Ophthalmol. 2014;157:433-.e3.

13. Beltran-Agullo L, Trope GE, Jin YP, Wagschal LD, Jinapriya D, Buys YM. Comparison of visual recovery following Ex-PRESS versus trabeculectomy: results of a prospective randomized controlled trial. J Glaucoma. 2015;24:181-6.

14. Chen G, Li W, Jiang F, Mao S, Tong Y. Ex-PRESS implantation versus trabeculectomy in open-angle glaucoma: a meta-analysis of randomized controlled clinical trials. PLoS ONE. 2014;9:e86045.

15. Law SK, Shih K, Tran DH, Coleman AL, Caprioli J. Long-term Outcomes of repeat vs initial trabeculectomy in open-angle glaucoma. Am J Ophthalmol. 2009;148:685-e1.

16. Yamana H, Moriwaki M, Horiguchi H, Kodan M, Fushimi K, Yasunaga $\mathrm{H}$. Validity of diagnoses, procedures, and laboratory data in Japanese administrative data. J Epidemiol. 2017;27: 476-82.

17. Law SK, Hosseini H, Saidi E, Nassiri N, Neelakanta G, Giaconi $\mathrm{JA}$, et al. Long-term outcomes of primary trabeculectomy in diabetic patients with primary open angle glaucoma. Br J Ophthalmol. 2013;97:561-6.

18. Edmunds B, Bunce CV, Thompson JR, Salmon JF, Wormald RP. Factors associated with success in first-time trabeculectomy for patients at low risk of failure with chronic open-angle glaucoma. Ophthalmology. 2004;111:97-103.

19. Dally LG, Ederer F, Gaasterland D, Blackwell B, Van Veldhuisen $\mathrm{P}$, Allen RC, et al. The Advanced Glaucoma Intervention Study (AGIS): 11. Risk factors for failure of trabeculectomy and argon laser trabeculoplasty. Am J Ophthalmol. 2002;134:481-98.

20. Stein JD, Ruiz D, Belsky D, Lee PP, Sloan FA. Longitudinal rates of postoperative adverse outcomes after glaucoma surgery among medicare beneficiaries 1994 to 2005. Ophthalmology. 2008;115:1109-e7.

21. Saheb H, Gedde SJ, Schiffman JC, Feuer WJ. Outcomes of glaucoma reoperations in the tube versus trabeculectomy (TVT) study. Am J Ophthalmol. 2014;157:1179-e2.

22. VanderWeele TJ, Ding P. Sensitivity analysis in observational research: introducing the E-value. Ann Intern Med. 2017;167:268.

23. Newhouse JP, McClellan M. Econometrics in outcomes research: the use of instrumental variables. Annu Rev Public Health. 1998; 19:17-34.

24. Tan H, Norton EC, Gore JL, Miller DC. Long-term survival following partial vs radical nephrectomy among older patients with early-stage kidney. Cancer 2012;307:1629-35.

25. Martínez-camblor P, Staiger DO, Goodney PP, Malley AJO. Adjusting for bias introduced by instrumental variable estimation in the Cox proportional hazards model. Biostatistics. 2019;20: 80-96. 
26. Urushiyama $H$, Jo $T$, Yasunaga $H$, Michihata $N$, Matsui $H$, Hasegawa W, et al. Oral fluorouracil vs vinorelbine plus cisplatin as adjuvant chemotherapy for stage II-IIIA non-small cell lung cancer: Propensity score-matched and instrumental variable analyses. Cancer Med. 2018;7:4863-69.

27. Broderick CR, Herbert RD, Latimer J, Barnes C, Curtin JA, Mathieu E, et al. Association between physical activity and risk of bleeding in children with hemophilia. JAMA. 2012; 308:1452.

28. Isogai T, Yasunaga H, Matsui H, Tanaka H, Horiguchi H, Fushimi K. Effectiveness of inferior vena cava filters on mortality as an adjuvant to antithrombotic therapy. Am J Med. 2015;128:312.e23-31.

29. Terza JV, Basu A, Rathouz PJ. Two-stage residual inclusion estimation: addressing endogeneity in health econometric modeling. J Health Econ. 2008;27:531-43.

30. Husain R, Clarke JCK, Seah SKL, Khaw PT. A review of trabeculectomy in East Asian people-the influence of race. Eye. 2005;19:243-52.

31. Kim S, Choi TH, Liu W, Ogawa R, Suh JS, Mustoe TA. Update on scar management: guidelines for treating asian patients. Plast Reconstr Surg. 2013;132:1580-9.

32. Ibrahim M, Bond J, Medina MA, Chen L, Quiles C, Kokosis G, et al. Characterization of the foreign body response to common surgical biomaterials in a murine model. Eur J Plast Surg. 2017; 40:383-92.

33. Qin B, Tang M, Li Y, Zhang X, Chu R, Huang D. Anterior segment dimensions in Asian and Caucasian eyes measured by optical coherence tomography. Ophthalmic Surg Lasers Imag. 2012;43:135-42.

34. Patel HY, Wagschal LD, Trope GE, Buys YM. Economic analysis of the Ex-PRESS miniature glaucoma device versus trabeculectomy. J Glaucoma. 2014;23:385-90.

35. Valentine J, Zurakowski D, Ayyala RS. Comparison of acquisition costs of surgical supplies in different health care systems for cataract and glaucoma procedures. J Glaucoma. 2014;23:355-9.

36. Hulsman CAA, Westendorp ICD, Ramrattan RS, Wolfs RCW, Witteman JCM, Vingerling JR, et al. Is open-angle glaucoma associated with early menopause? The rotterdam study. Am J Epidemiol. 2001;154:138-44.

37. Pasquale LR, Rosner BA, Hankinson SE, Kang JH. Attributes of female reproductive aging and their relation to primary open-angle glaucoma: a prospective study. J Glaucoma. 2007;16:598-605.

38. Friedman DS, Jampel HD, Lubomski LH, Kempen JH, Quigley $\mathrm{H}$, Congdon N, et al. Surgical strategies for coexisting glaucoma and cataract: an evidence-based update. Ophthalmology. 2002; 109:1902-15. 